\title{
A RELATIONSHIP BETWEEN THE LIPSCHITZ CONSTANTS APPEARING IN TAYLOR'S FORMULA
}

\author{
IOANNis K. ARgYros a AND Hongmin ReN ${ }^{\mathrm{b}}$
}

\begin{abstract}
Taylor's formula is a powerful tool in analysis. In this study, we assume that an operator is m-times Fréchet-differentiable and satisfies a Lipschitz condition. We then obtain some Taylor formulas using only the Lipschitz constants. Applications are also provided.
\end{abstract}

\section{INTRODUCTION}

Taylor's formula has been used for a long time as a powerful tool in analysis to study the convergence of iterative processes but also in other areas [1]-[4].

In this study we assume that operator $F$ is m-times ( $m$ a natural number) Fréchetdifferentiable on a non-empty subset $D$ of a Banach space $X$ with values in a Banach space $Y$. Furthermore, we assume that operator $F^{(m)}$ is Lipschitz continuous on $D$. Then, the constant is used to relate the corresponding Lipschitz constants for operators $F^{(i)}, i=1,2, \ldots, m$. Applications are also provided in this study.

\section{TAYlor Formulas}

We need the following results on Taylor's formula for $m$-Fréchet-differentiable operators.

Theorem 2.1. Let $G: D \subseteq X \rightarrow Y$ be a m-times $(m \in N)$ Fréchet-differentiable operators defined on a non-empty subset $D$ of a Banach space $X$ with values in a Banach space $Y$.

Assume: (a) there exist a constant $e_{m+1}>0$, and a convex subset $D_{0}$ of $D$ such that for all $x, y \in D_{0}$

$$
\left\|G^{(m)}(x)-G^{(m)}(y)\right\| \leq e_{m+1}\|x-y\|_{X} .
$$

Received by the editors August 18, 2011. Revised November 3, 2011. Accepted Nov. 8, 2011. 2000 Mathematics Subject Classification. 65G99, 47H17, 49M15.

Key words and phrases. Taylor's formula, Fréchet-differentiable operator, iterative processes, Banach space. 
Then, for all $x, y \in D_{0}$, the following estimate holds:

$$
\left\|G(x)-G(y)-\sum_{i=1}^{m} \frac{G^{(i)}(y)}{i !}(x-y)^{i}\right\|_{Y} \leq \frac{e_{m+1}}{(m+1) !}\|x-y\|_{X}^{m+1} .
$$

(b) If $D_{0}=U\left(x_{0}, R\right)=\left\{x \in X:\left\|x-x_{0}\right\| \leq R\right\} \subseteq D$ for some $x_{0} \in D, R>0$, and

$$
\left\|G^{(m)}(x)-G^{(m)}\left(x_{0}\right)\right\| \leq e_{m+1}^{0}\left\|x-x_{0}\right\|_{X} \quad \text { for some } e_{m+1}^{0}>0
$$

holds true on $D_{0}$, then

$$
\left\|G^{(k)}(x)\right\| \leq e_{k} \quad k=0,1, \ldots, m
$$

and

$$
\left\|G^{(k-1)}(x)-G^{(k-1)}(y)\right\| \leq e_{k}\|x-y\|_{X}, \quad k=1, \ldots, m,
$$

where, $e_{m}=\left\|G^{(m)}\left(x_{0}\right)\right\|+e_{m+1}^{0} R$,

$$
e_{k}=\left\|G^{(k)}\left(x_{0}\right)\right\|+e_{k+1}^{0} R, \quad k=0,1, \ldots, m-1 .
$$

(c) Under hypotheses of part (b) the following hold for all $x, y \in D_{0}$, and $k=$ $1,2, \ldots, m$ :

$$
\left\|G(x)-G(y)-\sum_{i=1}^{k} \frac{G^{(i)}(y)}{i !}(x-y)^{i}\right\|_{Y} \leq \frac{e_{k+1}}{(k+1) !}\|x-y\|_{X}^{k+1} .
$$

Proof. (a) Let us denote by $\alpha \in Y$ the element given by

$$
\alpha=G(x)-G(y)-\sum_{i=1}^{m} \frac{G^{(i)}(y)}{i !}(x-y)^{i} .
$$

It is well known [3], [4] that there exists $\beta \in L(Y, \mathbf{R})$ the space of bounded linear operators from $Y$ into $\mathbf{R}$ so that

$$
\|\beta\|_{X}=1, \quad \text { and } \beta(\alpha)=\|\alpha\|_{Y} .
$$

It then follows from (2.8), and (2.9) that

$$
\beta(\alpha)=\left|\beta(G(x))-\beta(G(y))-\sum_{i=1}^{m} \frac{\beta\left(G^{(i)}(y)(x-y)^{i}\right)}{i !}\right| .
$$

Let us define on $[0,1]$ the real function

$$
\gamma(\theta)=\beta(G(y+\theta(x-y))) .
$$


In view of the convexity of $D_{0}, y+\theta(x-y) \in D_{0}$ if $x, y \in D_{0}$. That is function $\gamma$ is well defined. It follows from the existence of the Fréchet-derivatives of operator $G$ that functions

$$
\gamma^{(k)}(\theta)=\beta\left(G^{(k)}(y+\theta(x-y))(x-y)^{k}\right) \quad k=0,1, \ldots, m
$$

are well defined.

Using the integral form of Taylor's formula [3], [4], we have:

$$
\gamma(1)=\gamma(0)+\sum_{i=1}^{m} \gamma^{(i)}(0)+\frac{1}{(m-1) !} \int_{0}^{1} \gamma^{(m)}(\theta)(1-\theta)^{p-1} d \theta .
$$

We also need the estimates:

$$
\int_{0}^{1}(1-\theta)^{m-1} d \theta=\frac{1}{m}
$$

and

$$
\int_{0}^{1} \theta(1-\theta)^{m-1} d \theta=\int_{0}^{1}(1-\theta)^{m-1} d \theta-\int_{0}^{1}(1-\theta)^{m} d \theta=\frac{1}{m}-\frac{1}{m+1}=\frac{1}{m(m+1)} .
$$

We then have:

$$
\begin{aligned}
\|\alpha\|_{Y} & =\frac{1}{(m-1) !}\left|\int_{0}^{1} \gamma^{(m)}(\theta)(1-\theta)^{m-1} d \theta-\int_{0}^{1} \gamma^{(m)}(0)(1-\theta)^{m-1} d \theta\right| \\
& \leq \frac{1}{(m-1) !} \int_{0}^{1}\left|\gamma^{(m)}(\theta)-\gamma^{(m)}(0)\right|(1-\theta)^{m-1} d \theta
\end{aligned}
$$

but

$$
\begin{aligned}
\left|\gamma^{(m)}(\theta)-\gamma^{(m)}(0)\right| & =\left|\beta\left(G^{(m)}(y+\theta(x-y))(x-y)^{m}\right)-\beta\left(G^{(m)}(y)\right)(x-y)^{m}\right| \\
& =\left|\beta\left(\left[G^{(m)}(y+\theta(x-y))(x-y)^{m}\right)-\left(G^{(m)}(y)\right]\right)(x-y)^{m}\right| \\
& \left.\leq\|\beta\| \| G^{(m)}(y+\theta(x-y))(x-y)^{m}\right)-\left(G^{(m)}(y)\|\| x-y \|_{X}^{m}\right.
\end{aligned}
$$

and consequently,

$$
\begin{aligned}
\|\alpha\|_{Y} & =\frac{e_{m+1}}{(m-1) !}\|x-y\|_{X}^{m+1} \int_{0}^{1} \theta(1-\theta)^{m-1} d \theta \\
& \leq \frac{e_{m+1}}{(m-1) ! m(m+1)}\|x-y\|_{X}^{m+1}
\end{aligned}
$$

That completes the proof of part (a).

(b) It follows from (2.3)

$$
\left\|G^{(m)}(x)\right\| \leq\left\|G^{(m)}\left(x_{0}\right)\right\|+e_{m+1}^{0}\left\|x-x_{0}\right\|=e_{m} .
$$

By Langrange's theorem applied to $G^{(m-1)}: D_{0} \rightarrow L\left(X^{m-1}, Y\right)$ we get

$$
\left\|G^{(m-1)}(x)-G^{(m-1)}(y)\right\| \leq\left\|G^{(m)}(y+\theta(x-y))\right\|\|x-y\|_{X} \quad \theta \in(0,1) .
$$


If $x, y \in D_{0}$, and $\theta \in(0,1)$ we get $y+\theta(x-y) \in D_{0}$ and consequently

$$
\left\|G^{(m)}(y+\theta(x-y))\right\| \leq e_{m},
$$

and

$$
\left\|G^{(m-1)}(x)-G^{(m-1)}(y)\right\| \leq e_{m}\|x-y\|_{X} .
$$

Estimates (2.3) and (2.4) are obtained by continuing the same way.

(c) This part follows immediately from parts (a), and (b).

That completes the proof of the theorem.

\section{Applications}

The first application involves the most popular iterative process which is Newton's method.

We need a result on majorizing sequences for Newton's method

$$
x_{n+1}=x_{n}-F^{\prime}\left(x_{n}\right)^{-1} F\left(x_{n}\right) \quad(n \geq 0),\left(x_{0} \in D\right)
$$

for generating a sequence $\left\{x_{n}\right\}$ approximating a solution $x^{\star}$ of equation

$$
F(x)=0 .
$$

Lemma 3.1 ([3, Lemma 1.1.2, p. 14]). Assume:

there exist constants $L_{0} \geq 0, L \geq 0$, with $L_{0} \leq L$, and $\eta \geq 0$, such that:

$$
q_{0}=\bar{L} \eta\left\{\begin{array}{l}
\leq \frac{1}{2}, \quad \text { if } \quad L_{0} \neq 0 \\
<\frac{1}{2}, \quad \text { if } \quad L_{0}=0
\end{array}\right.
$$

where,

$$
\bar{L}=\frac{1}{8}\left(L+4 L_{0}+\sqrt{L^{2}+8 L_{0} L}\right) .
$$

Then, sequence $\left\{t_{k}\right\}(k \geq 0)$ given by

$$
t_{0}=0, \quad t_{1}=\eta, \quad t_{k+1}=t_{k}+\frac{L\left(t_{k}-t_{k-1}\right)^{2}}{2\left(1-L_{0} t_{k}\right)} \quad(k \geq 1),
$$

is well defined, nondecreasing, bounded from above by $t^{\star \star}$ and converges to its unique least upper bound $t^{\star} \in\left[0, t^{\star \star}\right]$, where

$$
\begin{gathered}
t^{\star \star}=\frac{2 \eta}{2-\delta}, \\
1 \leq \delta=\frac{4 L}{L+\sqrt{L^{2}+8 L_{0} L}}<2 \text { for } L_{0} \neq 0 .
\end{gathered}
$$


Moreover, the following estimates hold:

$$
\begin{gathered}
L_{0} t^{\star} \leq 1, \\
0 \leq t_{k+1}-t_{k} \leq \frac{\delta}{2}\left(t_{k}-t_{k-1}\right) \leq \cdots \leq\left(\frac{\delta}{2}\right)^{k} \eta, \quad(k \geq 1), \\
t_{k+1}-t_{k} \leq\left(\frac{\delta}{2}\right)^{k}\left(2 q_{0}\right)^{2^{k}-1} \eta, \quad(k \geq 0), \\
0 \leq t^{\star}-t_{k} \leq\left(\frac{\delta}{2}\right)^{k} \frac{\left(2 q_{0}\right)^{2^{k}-1} \eta}{1-\left(2 q_{0}\right)^{2^{k}}}, \quad\left(2 q_{0}<1\right), \quad(k \geq 0) .
\end{gathered}
$$

We also need the result related to Lemma 3.1.

Lemma 3.2. Let $m \geq 2$ be a natural number; $\alpha_{i}$ non-negative numbers, $i=$ $2, \ldots, m+1, \eta>0$ and define functions $P, \bar{L}_{0}, \bar{L}, H$ on $(0,+\infty)$ by

$$
\begin{gathered}
P(r)=\frac{\alpha_{m+1}}{(m+1) !} r^{m+1}+\frac{\alpha_{m}}{m !} r^{m}+\ldots+\frac{\alpha_{2}}{2 !} r^{2}-r+\eta \\
\bar{L}_{0}(r)=\frac{1+p^{\prime}(r)}{r}=\frac{\alpha_{m+1}}{m !} r^{m-1}+\frac{\alpha_{m}}{(m-1) !} r^{m-2}+\ldots+\alpha_{2}, \\
\bar{L}(r)=P^{\prime \prime}(r)=\frac{\alpha_{m+1}}{(m-1) !} r^{m-1}+\frac{\alpha_{m}}{(m-2) !} r^{m-2}+\ldots+\alpha_{2}
\end{gathered}
$$

and

$$
H(r)=\left(\bar{L}(r)+4 \bar{L}_{0}(r)+\sqrt{\bar{L}^{2}(r)+8 \bar{L}_{0}(r) \bar{L}(r)}\right) \eta-4
$$

Assume:

$$
H(\eta)<0
$$

Then, function $H$ has a unique positive zero $r_{0}$ such that

$$
r_{0}>\eta
$$

Moreover, for a fixed $r^{\star} \in\left(\eta, r_{0}\right]$, set

$$
L_{0}=\bar{L}_{0}\left(r^{\star}\right), \quad \text { and } \quad L=\bar{L}\left(r^{\star}\right) \text {. }
$$

Then, the conclusions of Lemma 3.1 hold for iteration $\left\{t_{n}\right\}$. 
Proof. Function $H$ is well defined, since $\bar{L}_{0}$, and $\bar{L}$ are positive functions. Moreover, $H$ is increasing, since $H^{\prime}(r)>0$ for $r>0$. It then follows from (3.12) that $H(r)>$ 0 for sufficiently large $r>0$. The existence, uniqueness of $r_{0}$ follows from the intermediate value theorem, and the monotonicity of function $H$, respectively. Using the definition of $L_{0}, L$, and $H$, we deduce that estimate (3.17) holds.

That completes the proof of lemma.

Hypothesis (3.16) can be replaced by the weaker, and more general

$$
\text { Function } H \text { has a minimal positive zero } r_{0} \text {. }
$$

We can show the following semilocal convergence result for Newton's method (3.1).

Proposition 3.3. Let $F: D \subseteq X \rightarrow Y$ be a m-times Fréchet-differentiable operator defined on a non-empty, open and convex subset $D$ of a Banach space $X$ with values in a Banach space $Y$. Assume there exists $x_{0} \in D, e_{m+1}>0$ such that

$$
\begin{gathered}
F^{\prime}\left(x_{0}\right)^{-1} \in D \\
\left\|F^{\prime}\left(x_{0}\right)^{-1}\left(F^{\prime}(x)-F^{\prime}(y)\right)\right\| \leq e_{m+1}\|x-y\| \quad \text { for all } x, y \in D ;
\end{gathered}
$$

hypotheses of Lemmas 2.1, 2.2 hold for

$$
\begin{gathered}
G=F^{\prime}\left(x_{0}\right)^{-1} F, \quad \alpha_{i}=e_{i} \quad i=1,2, \ldots, m+1 ; \\
\bar{U}\left(x_{0}, \alpha^{\star}\right) \subseteq D
\end{gathered}
$$

and

$$
\alpha^{\star}=t^{\star} \text { or } t^{\star \star}<r_{0}
$$

where $t^{\star}, t^{\star \star}$ are given in Lemma 3.1, and $r_{0}$ is in Lemma 3.2.

Then, sequence $\left\{x_{n}\right\}$ generated by Newton's method (3.1) is well-defined, remains in $\bar{U}\left(x_{0}, \alpha^{\star}\right)$ for all $n \geq 0$, and converges to a unique solution $x^{\star} \in \bar{U}\left(x_{0}, \alpha^{\star}\right)$ of equation $F(x)=0$.

Moreover, the following estimates hold for all $n \geq 0$ :

$$
\left\|x_{n+1}-x_{n}\right\| \leq t_{n+1}-t_{n}
$$

and

$$
\left\|x_{n}-x^{\star}\right\| \leq t^{\star}-t_{n} .
$$


Proof. Simply repeat the proof of Theorem by Argyros in [2], but use (2.2) for $x=x_{n+1}, y=x_{n}$ to obtain:

$$
\begin{gathered}
\left\|F^{\prime}\left(x_{n}\right)^{-1} F^{\prime}\left(x_{0}\right)\right\| \leq \frac{1}{1-L\left\|x_{n}-x_{0}\right\|} \leq \frac{1}{1-L t_{n}}, \\
\left\|F^{\prime}\left(x_{0}\right)^{-1} F^{\prime}\left(x_{n}\right)\right\| \leq \frac{L}{2}\left\|x_{n+1}-x_{n}\right\|^{2} \leq \frac{L}{2}\left(t_{n+1}-t_{n}\right)^{2},
\end{gathered}
$$

and by (3.1)

$$
\left\|x_{n+1}-x_{n}\right\| \leq\left\|F^{\prime}\left(x_{n}\right)^{-1} F^{\prime}\left(x_{0}\right)\right\| F^{\prime}\left(x_{0}\right)^{-1} F\left(x_{n}\right) \| \leq \frac{L\left(t_{n+1}-t_{n}\right)^{2}}{2\left(1-L_{0} t_{n}\right)}=t_{n+1}-t_{n} .
$$

That completes the proof of the proposition.

As a second application, consider $X=Y=\mathbf{R}, D=\bar{U}(0,1)$, and define function $F$ on $D$ by

$$
F(x)=e^{x}
$$

Then, for any $m \geq 1$,

(3.31) $a_{m+1}=e, \quad\left\|F^{\prime}\left(x_{0}\right)^{-1} F^{(m)}\left(x_{0}\right)\right\|=1, \quad a_{m+1}^{0}=e-1, \quad a_{m}=1+(e-1) R$, and

$$
a_{k}=1+e R, \quad k=0,1,2, \ldots, m-1 .
$$

Estimates (2.2) and (2.7) can now be obtained with these choices.

\section{REFERENCES}

1. I.K. Argyros: A Newton-Kantorovich theorem for equations involving m-Fréchet differentiable operators and applications in radiative transfer. J. Comput. Appl. Math. 131 (2001), 149-159.

2. ___ On the Newton-Kantorovich hypothesis for solving equations. J. Comput. Appl. Math. 169 (2004), 315-332.

3. _ : Advances on iterative procedures. Nova Science Publ., Inc., 2011.

4. L.M. Graves: Riemann integration and Taylor's theorem in general analysis. Trans. Amer. Math. Soc. 29 (1927), 163-177.

${ }^{a}$ Cameron university, Department of Mathematics Sciences, Lawton, OK 73505, USA

Email address: iargyros@cameron.edu

${ }^{\mathrm{b}}$ College of Information and Electronics, Hangzhou 311402, Zhejiang, P. R. China

Email address: rhm65@126.com 